\title{
Cryogenic Propellant Feed System Analytical Tool Development
}

\author{
Brian S. Lusby ${ }^{1}$ \\ NASA Johnson Space Center, Houston, TX, 77058 \\ Bruno M. Miranda ${ }^{2}$ \\ Engineering and Science Contract Group, Houston, TX, 77258 \\ and \\ Jacob A. Collins ${ }^{3}$ \\ NASA Johnson Space Center, Houston, TX, 77058
}

The Propulsion Systems Branch at NASA's Lyndon B. Johnson Space Center (JSC) has developed a parametric analytical tool to address the need to rapidly predict heat leak into propellant distribution lines based on insulation type, installation technique, line supports, penetrations, and instrumentation. The Propellant Feed System Analytical Tool (PFSAT) will also determine the optimum orifice diameter for an optional thermodynamic vent system (TVS) to counteract heat leak into the feed line and ensure temperature constraints at the end of the feed line are met. PFSAT was developed primarily using Fortran 90 code because of its number crunching power and the capability to directly access real fluid property subroutines in the Reference Fluid Thermodynamic and Transport Properties (REFPROP) Database developed by NIST. A Microsoft Excel front end user interface was implemented to provide convenient portability of PFSAT among a wide variety of potential users and its ability to utilize a user-friendly graphical user interface (GUI) developed in Visual Basic for Applications (VBA). The focus of PFSAT is on-orbit reaction control systems and orbital maneuvering systems, but it may be used to predict heat leak into ground-based transfer lines as well. PFSAT is expected to be used for rapid initial design of cryogenic propellant distribution lines and thermodynamic vent systems. Once validated, PFSAT will support concept trades for a variety of cryogenic fluid transfer systems on spacecraft, including planetary landers, transfer vehicles, and propellant depots, as well as surface-based transfer systems. The details of the development of PFSAT, its user interface, and the program structure will be presented.

\section{Nomenclature [need to update]}

$\begin{array}{ll}\mathrm{LCH}_{4} & =\text { Liquid Methane } \\ \mathrm{LO}_{2} & =\text { Liquid Oxygen } \\ \mathrm{A} & =\text { amplitude of oscillation }\end{array}$

\section{Acronyms}

$\begin{array}{ll}\text { CFM } & =\text { Cryogenic Fluid Management } \\ \text { CPST } & =\text { Cryogenic Propulsion Storage and Transfer } \\ \text { DLL } & =\text { Dynamic Link Library } \\ \text { ESEOD } & =\text { Equivalent Sharp Edge Orifice Diameter } \\ \text { ESTA } & =\text { Energy Systems Test Area }\end{array}$

${ }_{1}^{1}$ Engineer, Propulsion Systems Branch, 2101 NASA Parkway/EP4, Houston, TX 77058.

${ }^{2}$ Analyst, Thermal Analysis Group, 2224 Bay Area Boulevard, Houston, TX 77258.

${ }^{3}$ Project Manager, Propulsion Systems Branch, 2101 NASA Parkway/EP4, Houston, TX 77058. 


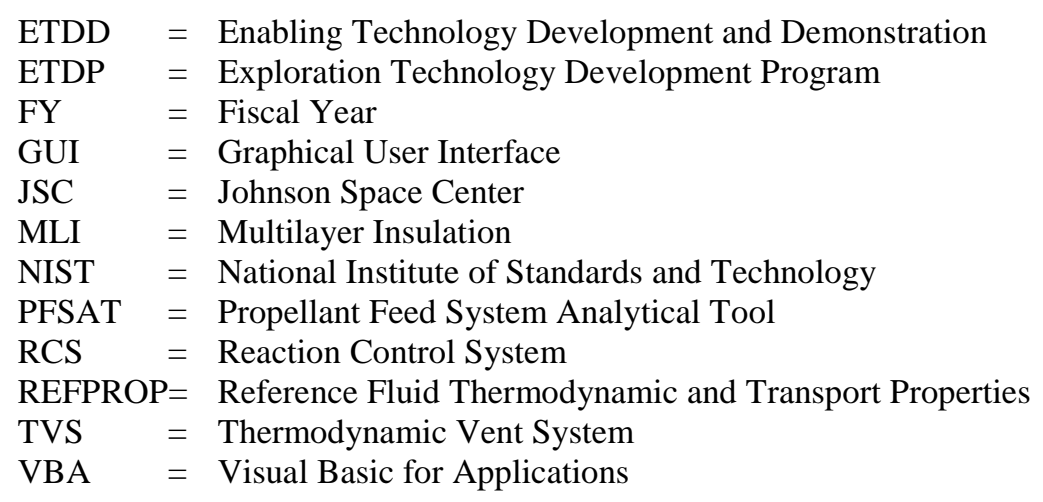

\section{Introduction}

$\mathrm{T}_{\mathrm{P}}^{\mathrm{H}}$ HE Cryogenic Fluid Management (CFM) Project under NASA's former Exploration Technology Development Program (ETDP) identified a need for an analytical tool for cryogenic feed systems in order to predict heat leak into propellant transfer lines based on insulation type, installation technique, line supports, penetrations, and instrumentation. Initial development of the Propellant Feed System Analytical Tool (PFSAT) began during fiscal year (FY) 2010 and has continued through FY 2011 under the auspices of the Cryogenic Propulsion Storage and Transfer (CPST) Project under the newly formed Enabling Technology Development and Demonstration (ETDD) Program. PFSAT has been designed to predict heat leak into spacecraft cryogenic main stage and reaction control propulsion systems, but it may be used to predict heat leak into ground-based transfer lines as well. The Cryogenic Feed System Test Bed at Johnson Space Center (JSC) [insert references] is being used as the baseline configuration for PFSAT to model. The Cryogenic Feed System Test Bed is a full scale reaction control system (RCS) feed line with integral thermodynamic vent system (TVS) and high performance multilayer insulation (MLI) located inside a 15 foot thermal vacuum chamber. The data obtained from this test program is currently being used to validate the tool. Once validated, PFSAT will be used to support concept trade studies and initial design of propellant distribution lines and thermodynamic vent systems.

PFSAT has been developed primarily as Fortran 90 code with a Microsoft Excel/Visual Basic for Applications (VBA) front end user interface. Fortran 90 was selected as the primary coding language because of its powerful number crunching ability. In addition, it was desirable to maintain consistency with other CFM analytical tools that were developed using FORTRAN code in preparation for the possible future integration of PFSAT with one or more of these tools [insert references]. Microsoft Excel was chosen as the tool's front end due to the commonality of the software and its ability to utilize a user-friendly graphical user interface (GUI) developed in VBA. VBA is also used to access the Fortran 90 functions and subroutines that comprise the heart of the tool through compiled dynamic link libraries (DLL). This chosen architecture provides convenient portability of PFSAT among a wide variety of potential users.

\section{System Description}

The primary objective of PFSAT is to predict heat leak into cryogenic propellant distribution lines with specific applications to spacecraft propulsion systems. The JSC Propulsion Systems Branch has been conducting research and development of liquid oxygen $\left(\mathrm{LO}_{2}\right)$ / liquid methane $\left(\mathrm{LCH}_{4}\right)$ propulsion technology for the past several years, and in particular several phases of testing on the Cryogenic Feed System Test Bed have been completed since 2005 [insert references]. The Cryogenic Feed System Test Bed is a full scale RCS feed line with integral TVS, high performance MLI, and flight-like line supports located inside the 15 foot thermal vacuum chamber at JSC's Energy Systems Test Area (ESTA). Accordingly, PFSAT has been designed based on this test bed configuration, but its construct has been generalized to allow parametric analysis capabilities. Figure 1 below shows a generic representation of a system based on the ESTA test bed that PFSAT has been designed to analyze. 


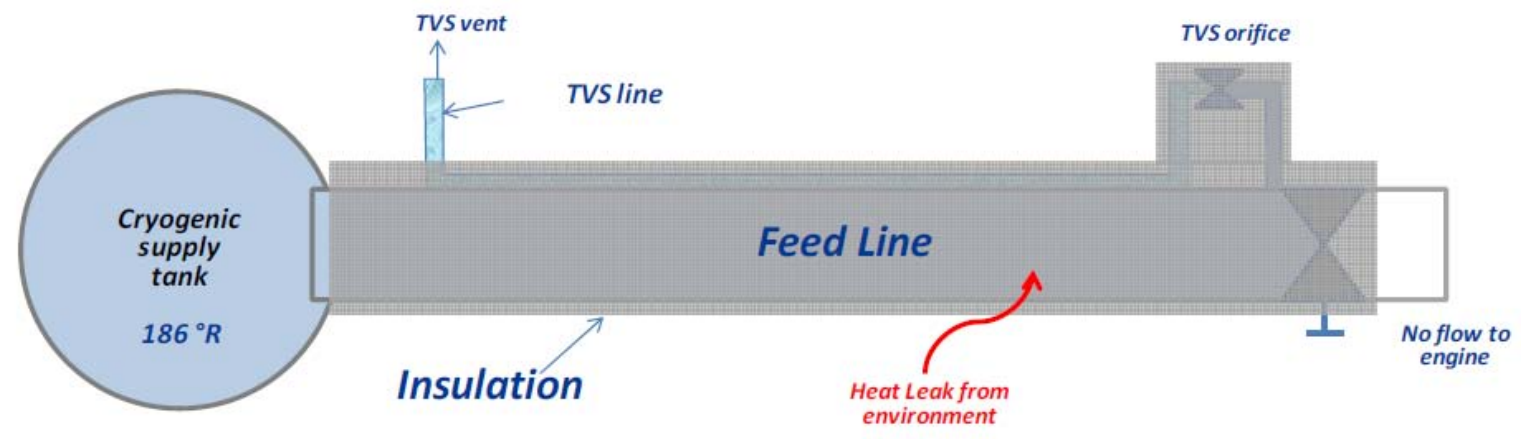

Figure 1. Generic system representation.

PFSAT models the propellant feed line from the propellant tank outlet to the engine valve inlet. The upstream boundary condition is specified by the user as a fixed temperature and pressure, which represents the propellant tank outlet. At the end of the propellant feed line are one or more rocket engines. It has been shown that during periods of high engine duty cycle, the TVS is not needed because engine usage is enough to reject the energy gained due to heat transfer from the environment and keep the feed line chilled in [insert reference]. Therefore, PFSAT is designed for periods of low duty cycle or quiescent mode operation when the TVS is needed in order to keep the feed line chilled in and ready for operation. The TVS serves as a method of rejecting heat from the feed line by JouleThomson expansion and subsequent boiloff of a very small quantity of propellant. This is accomplished by throttling the propellant at the termination of the feed line through a TVS valve with an extremely small flow area thereby decreasing the propellant temperature to its saturation point, and then routing the TVS line back along the feed line to create a counterflow heat exchanger removing sensible heat from the feed line propellant via latent heat absorbed and rejected by the two-phase fluid in the TVS line.

In order to size the TVS valve flow area, the user must specify the maximum engine inlet temperature. This is the temperature at the end of the feed line that would result from TVS usage during quiescent mode operation. PFSAT calculates the heat leak into the feed line based on the insulation parameters provided by the user and determines the TVS flow rate needed to achieve the required engine inlet temperature. Based on this flow rate, PFSAT will determine the required TVS valve equivalent sharp edge orifice diameter (ESEOD) to generate the cooling to achieve the required temperature profile. The result is a steady-state solution in which the TVS will provide indefinite cooling to the feed line such that the heat leak is appropriately balanced to maintain the required engine inlet temperature.

\section{User Interface}

Microsoft Excel with VBA was chosen as the front end user interface for PFSAT due to its commonality and portability among a wide variety of potential users. A flow chart of PFSAT and its color-coded worksheets is shown below in Fig. 2. When PFSAT is opened the "PFSAT Setup" worksheet, color-coded green, is displayed. As its name implies, this worksheet is used to setup a particular simulation and initialize the solver. In order to control the configuration of the workbook, this worksheet is protected so the cells cannot be modified and the various inputs are collected from the user through a series of VBA input forms, which are accessed through the button labeled "Get Inputs from User". The inputs obtained from the user are then displayed on the worksheet for easy reference. The solver is initiated when the user clicks the "Run TVS Solver" button. Once the solver converges on a solution, the results are populated into three worksheets, color-coded blue. PFSAT also generates six charts to graphically represent the results in English units, color-coded yellow, and SI units, color-coded pink. 


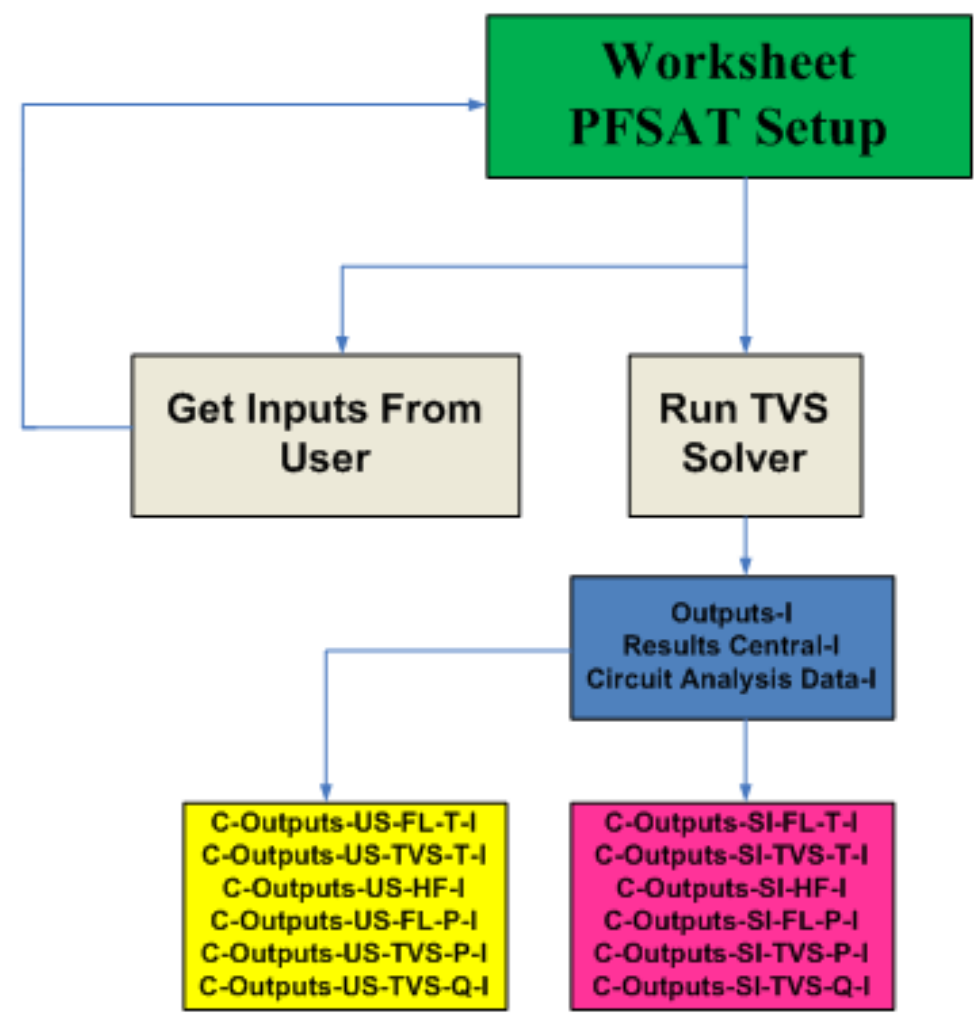

Figure 2. PFSAT flow chart.

The complete set of inputs required by PFSAT to fully define the system and analysis conditions has been divided into eight VBA input forms to keep similar inputs grouped together on separate forms. When the user clicks the button labeled "Get Inputs from User" from the "PFSAT Setup" worksheet, the main inputs menu shown in Fig. 3 is activated, which contains buttons to access each of the eight input forms. The information entered through these forms is passed to the equivalent location on the "PFSAT Setup" worksheet where they will be used to initialize the solver.

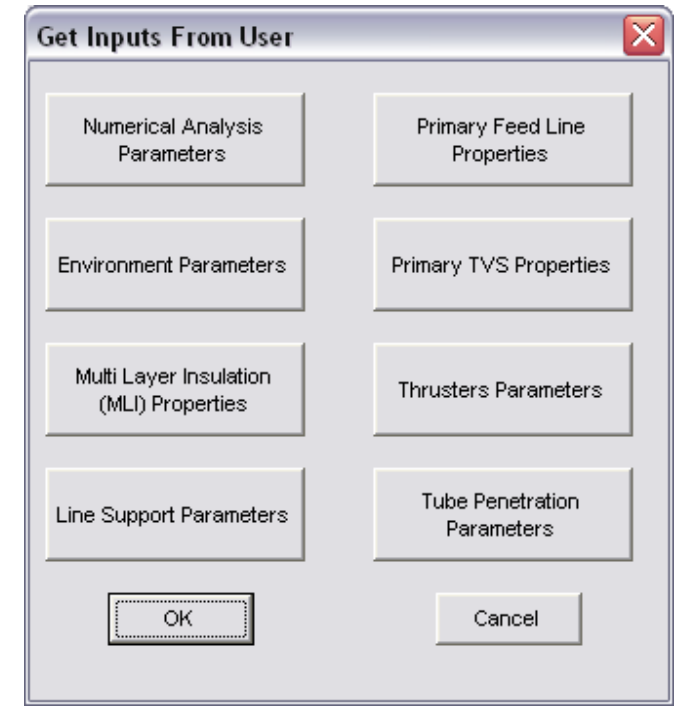

Figure 3. Main inputs menu.

The "Numerical Analysis Parameters" button brings up an input form in which the user can input basic parameters that control the performance of the solver. The "Environment Parameters" button brings up an input

American Institute of Aeronautics and Astronautics 
form in which the user is asked to input the environment boundary conditions. To be as flexible as possible, separate inputs for the radiation source temperature and the atmosphere temperature have been included so that radiation and convection can be modeled separately if needed. Since it is intended to apply this tool to ground transfer lines in addition to spacecraft, the inclusion of external free convection is warranted. Consequently, there is also an input for environment pressure and fluid. These inputs are also applicable to the MLI heat leak calculations for cases in which the vacuum environment is greater than $10^{-6}$ torr when interstitial gas conduction between the insulation layers cannot be neglected [insert reference].

Figure 4 shows the input form that is activated when the user clicks the "Primary Feed Line Properties" button. This figure is an example of one of the eight input forms accessed from the main inputs menu. The first few inputs on the primary feed line input form are the propellant being considered and the temperature and pressure at which the fluid is being stored in the propellant tank. Available fluids are the cryogenic propellants oxygen, hydrogen, and methane. Nitrogen is also available as it can be used as a cryogenic propellant simulator for safe thermal performance test systems, including the baseline test bed that is being used to validate PFSAT. Next the user inputs the feed line geometric parameters and the tubing material. The tube outer diameter and wall thickness inputs have been limited to commercially available sizes in $/ 8$ inch increments up to two inch outer diameter. Tubing materials available for selection include stainless steel 304 and 316 as well as titanium alloys Ti-3Al-2.5V and Ti-6Al-4V. Finally, the user is asked to input the degrees of subcooling at the end of the feed line. This input represents the engine valve inlet temperature requirement, which is used to size the TVS flow area based on the calculated heat leak.

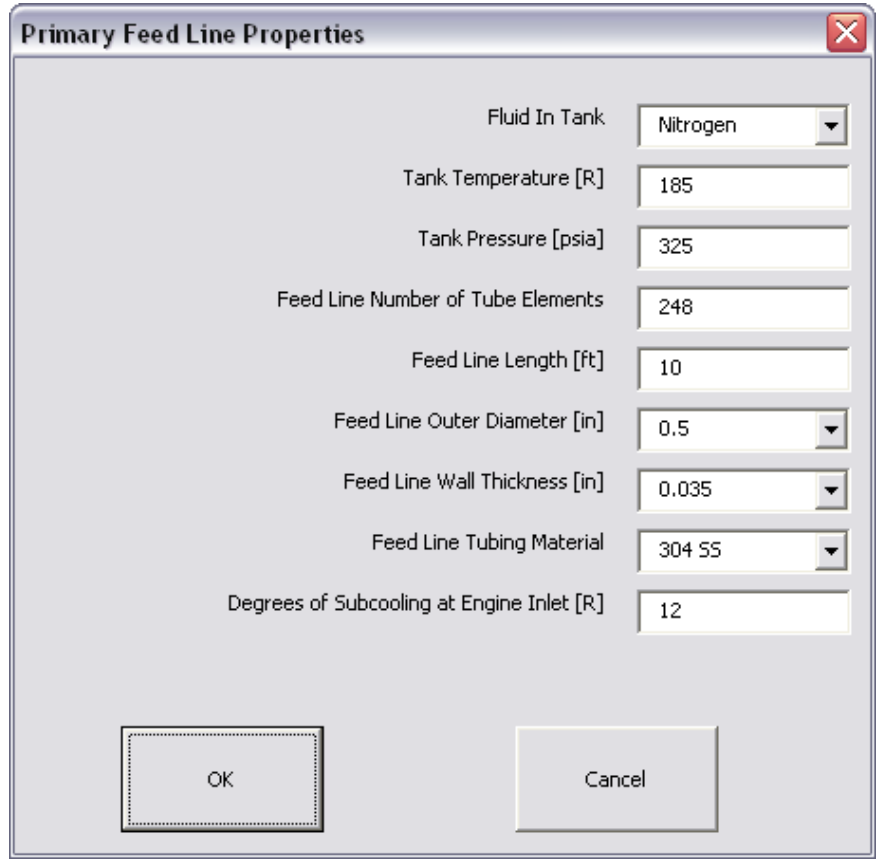

Figure 4. Primary feed line properties input form.

An input for the number of elements along the feed line is available for the user to control the amount of discretization. The input value should be high enough so that the results generated are independent of the number of elements. If the results for a given number of elements show only slight variances while using 1.1 times that number of elements, the grid is considered suitable. A minimum of nine elements is required because four small elements are automatically used at the entrance and exit of the feed line. However, if the user chooses to consider heat leak from line supports in the analysis, PFSAT will automatically update the number of elements such that the length of the element is determined by the dimensions of supports.

Similar to the primary feed line inputs, the "Primary TVS Properties" button activates an input form that allows the user to specify the TVS line geometric parameters and tubing material. Again the tube outer diameter and wall thickness inputs have been limited to commercially available sizes. The length of the TVS line is assumed to be the same as the feed line so that it can be discretized in the same manner. Also specified by the user on this form is the method by which the TVS line is thermally coupled to the feed line, which can include various thermal epoxy or 
braze materials or simple hose clamps. This information is used to calculate the conductance between the feed line and the TVS line. Finally, the user must also input the expected back pressure at the end of the TVS line as the downstream boundary condition required for flow rate calculations.

The "Multilayer Insulation (MLI) Properties" button activates the input form shown in Fig. 5. The preferred method of calculating heat leak through the MLI is the Lockheed relation developed in Ref. 1, but flexibility has been incorporated into PFSAT for other methods to be considered. The input form contains a dropdown menu for the user to select which method is to be used. The first option is simply a user-defined effective emissivity, $\varepsilon^{*}$, to be used in the radiation heat transfer rate equation. This is the most straightforward method of data entry available for the MLI properties. This can be useful when this value is known from test data and the user wants to examine the effects of other system parameters on the overall system performance. The second option uses the true emissivity of each side of the radiation shield material and the number of layers to estimate the effective emissivity. This is a purely theoretical method to calculate the ideal emittance, $\varepsilon$, for highly evacuated MLI systems with non-contacting layers and interstitial gas pressure less than $10^{-5}$ torr [insert reference]. The values computed using this method represent a best case scenario with the lowest possible MLI heat leak. The next option is the Doenecke relation, which is an empirical extension of the true emissivity theory [insert reference]. The Doenecke relation relates the effective emittance, $\varepsilon_{\text {eff }}$, to the number of layers, the blanket size, the percent open area (perforations), and the average temperature of the blankets. The final option to calculate heat leak though the MLI is the Lockheed relation, which is an empirical method discussed in more detail later.

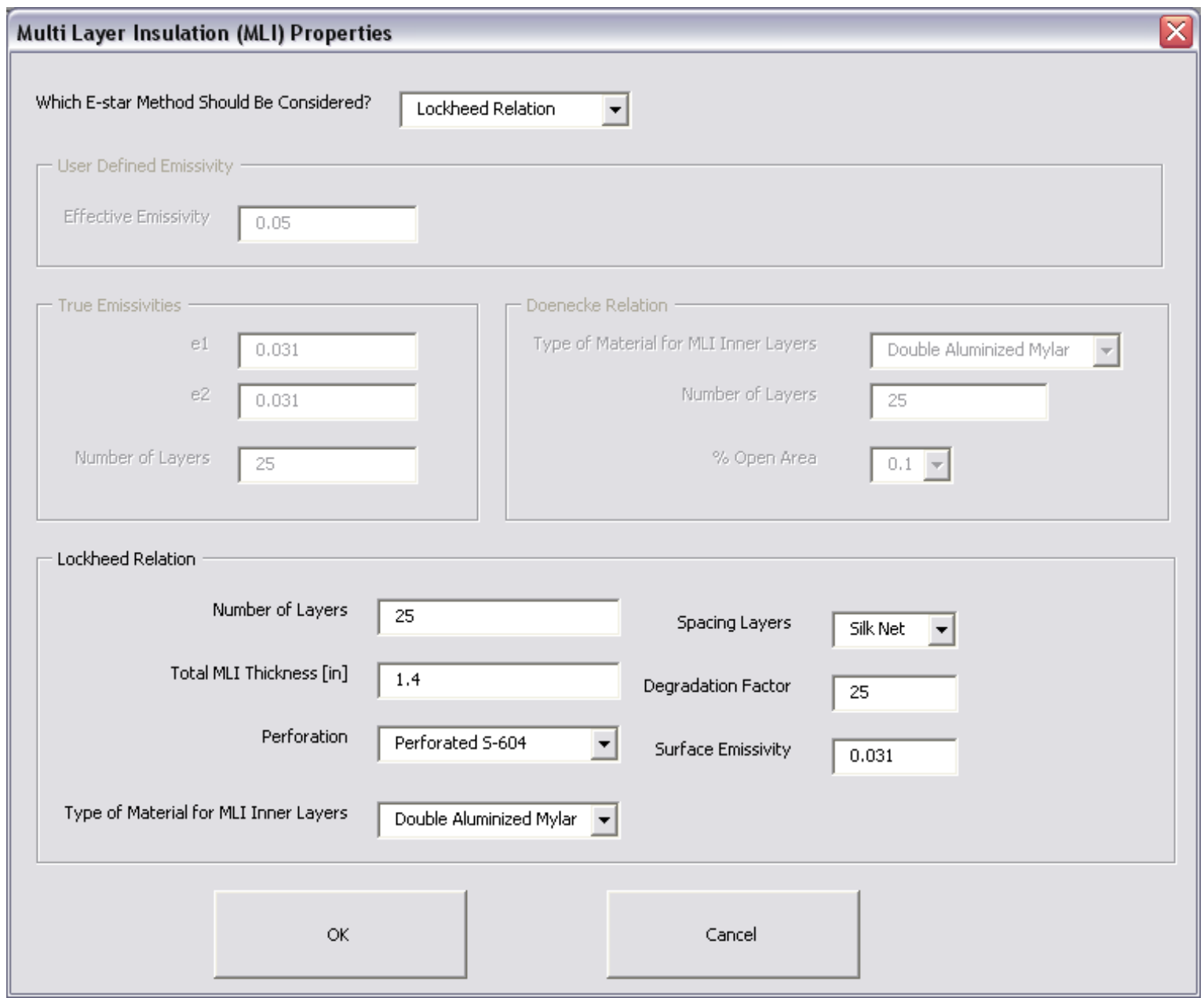

Figure 5. Multilayer insulation input form with the Lockheed relation selected.

Heat leak contributions due to feed line supports and penetrations are both modeled in a similar manner, thus, requiring comparable inputs via the "Line Support Parameters" and "Tube Penetration Parameters" buttons, respectively. Hot side boundary temperature inputs for the line supports and tube penetrations are independent of 
each other to provide full parametric capability, although it is usually sufficient to use the same temperature as the environment. Line support materials available for selection include various formulations of Ultem resin and G-10 glass. Tube penetration materials available for selection are the same as those available for the feed line and TVS tubing. The geometry of the line supports is based on the supports that were used in the ESTA Cryogenic Feed System Test Bed, a diagram of which is shown in Fig. 6. PFSAT will automatically scale the geometry based on the feed line diameter supplied by the user. The tube penetrations geometric inputs are similar to that of the feed line and TVS line, which includes the length, outer diameter, and tube wall thickness. An additional option for tube penetrations is given to model instrumentation as a solid rod in which only the length and outer diameter are required to specify the geometry. Finally, the user is asked to input the number of line supports and penetrations, and the locations are specified such that they are either equally spaced along the feed line or the distances from the feed line entrance are input manually. The user also has the option to turn off consideration of each of these heat leak contributors so that only the MLI is considered.

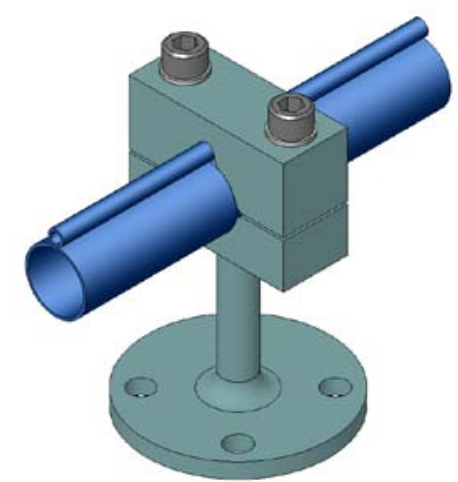

Figure 6. Line support geometry. [Can I use this picture from Ball Aerospace \& Technologies Corp.?]

After the user has populated all the required inputs, the solver is initiated by clicking the "Run TVS Solver" button. Once the solver converges on a solution, the results in English and SI units are populated into the three worksheets with blue color-coded tabs. The "Outputs-I" worksheet is populated with a detailed listing of temperatures, pressures, vapor quality, and heat flux as a function of distance along the feed line and TVS line. The "Results Central-I" worksheet is populated with a summary of the key results that the user would be most interested in, such as the total feed line heat leak, TVS flow rate, TVS valve pressure drop and ESEOD, and the heat removed by the TVS. The "Circuit Analysis Data-I" worksheet is populated with the detailed set of thermal resistance and heat flow results from the thermal circuit analysis described in the next section. This worksheet is provided for detailed examination of the results generated by the solver for advanced design and troubleshooting purposes. Using the results populated in the "Outputs-I worksheet, PFSAT generates six charts to graphically represent the results. These charts include the temperature gradients along the feed line and TVS line, the heat flux along the feed line and TVS line, the pressure gradients along the feed line and TVS line, and the vapor quality along the TVS line. Each chart is displayed in both English and SI units.

\section{Theory}

The propellant feed line with coupled TVS is modeled similar to a counterflow heat exchanger in terms of its equivalent electrical circuit using the finite difference method. The flow network is discretized into small elements of length $D x$ so that the thermal circuit can be solved for the system's steady-state temperature gradients. Figure 7 shows the thermal circuit representation of a single element, $j$, of the counterflow heat exchanger. The propellant feed line is considered to contain the hot fluid in the heat exchanger, represented by variables with subscript $H$, while the TVS line contains the cold fluid, represented by variables with the subscript $C$. Each element of each fluid line is represented by two nodes: a node for the tube wall temperature, with subscript $W$, and a node for the fluid temperature, with subscript $F$. The feed line element is thermally coupled to the TVS line element through thermal resistance $R 5$, which represents the thermal resistance of the material used to bond the two lines together. 

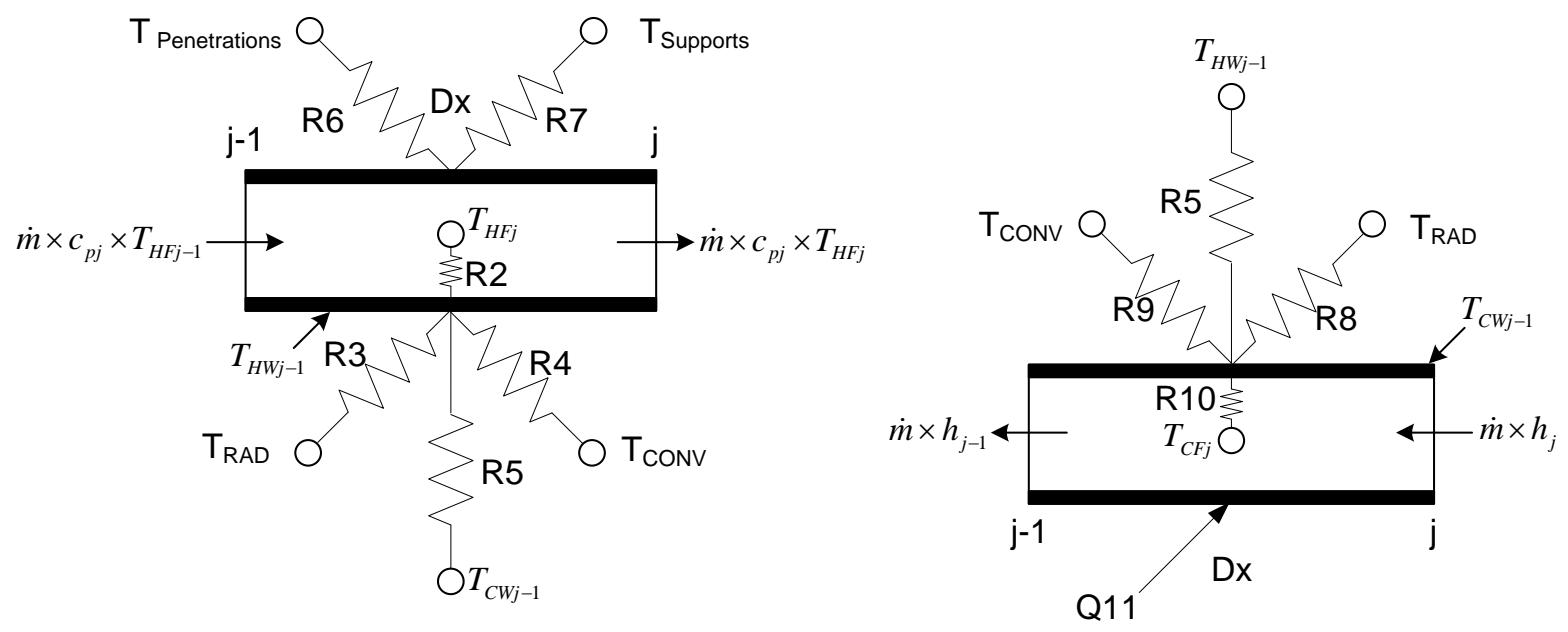

Figure 7. Thermal circuit representation of element $j$ of the counterflow heat exchanger.

The various thermal resistances shown in Fig. 7 can be calculated based on the inputs obtained from the user. Each tube wall temperature node is connected to two thermal resistors from the environment: one for radiation heat transfer and one for convection heat transfer. Similarly, each tube wall temperature node is connected to thermal resistors representing conduction from line supports and penetrations. PFSAT has been configured such that the user has the freedom to enter separate boundary node temperatures for the radiation and convective environments as well as the penetrations and supports to provide full parametric capability.

Energy balances can be written for feed line nodes $T_{H F j}$ and $T_{H W j-1}$ and are shown in Eqs. (1) and (2). Substituting the appropriate equations for the thermal resistances due to conduction, convection, and radiation shown in Fig. 7 and rearranging Eqs. (1) and (2) results in two equations with two unknowns. However, Eq. (2) is nonlinear, and therefore must be linearized in order to be iteratively solved. A trial value of $T_{H W j-1}$ is used to begin the iteration process until the convergence criterion is met. Once Eq. (2) is solved, the resulting value of $T_{H W j-1}$ can be used to solve for $T_{H F j}$ in Eq. (1). The complete derivation of the solution algorithm is shown in the PFSAT User's Guide [insert reference].

$$
\begin{gathered}
\frac{T_{H F j}}{R 1}=\frac{T_{H F j-1}}{R 1}+\frac{\left(T_{H W j-1}-T_{H F j}\right)}{R 2} \\
\frac{\left(T_{H W j-1}-T_{H F j}\right)}{R 2}+\frac{\left(T_{H W j-1}-T_{C W j-1}\right)}{R 5}= \\
+\frac{\left(T_{R A D}-T_{H W j-1}\right)}{R 3}+\frac{\left(T_{C O N V}-T_{H W j-1}\right)}{R 4} \\
+\frac{\left(T_{\text {Penetaration }}-T_{H W j-1}\right)}{R 6}+\frac{\left(T_{\text {Support }}-T_{H W j-1}\right)}{R 7}
\end{gathered}
$$

Where [define terms]

Similarly, energy balances for TVS line nodes $T_{C F j}$ and $T_{C W j-1}$ are shown in Eqs. (3) and (4). Again, substituting the appropriate equations for the thermal resistances shown in Fig. 7 and rearranging Eqs. (3) and (4) results in two equations with two unknowns. Equation (4) is nonlinear, and therefore must be linearized in order to be iteratively solved similar to how the feed line wall temperature node was solved. A trial value of $T_{C W j-1}$ is used to begin the iteration process until the convergence criterion is met. Once Eq. (4) is solved, the resulting value of $T_{C W j-1}$ can be used to solve for $T_{C F j}$ in Eq. (3). Again, the complete derivation of the solution algorithm is shown in the PFSAT User's Guide [insert reference]. 


$$
\begin{gathered}
h_{j-1}=h_{j}+\frac{\left(T_{C W j-1}-T_{C F j}\right)}{R 10} \times \frac{1}{\dot{m}} \\
\frac{\left(T_{C W j-1}-T_{C F j}\right)}{R 10}=\frac{\left(T_{H W j-1}-T_{C W j-1}\right)}{R 5}+\frac{\left(T_{R A D}-T_{C W j-1}\right)}{R 8}+\frac{\left(T_{C O N V}-T_{C W j-1}\right)}{R 9}+Q 11
\end{gathered}
$$

Where [define terms]

The Reference Fluid Thermodynamic and Transport Properties (REFPROP) standard reference database ${ }^{2}$, developed by the National Institute of Standards and Technology (NIST), has been incorporated into PFSAT as a means to calculate the real fluid thermodynamic and transport properties during the solution algorithm. The use of REFPROP provides PFSAT with the capability to update fluid properties for each discrete element in the system during every iteration as the trial temperatures change in order to reach convergence. This ensures that accurate real fluid properties are used to find the solution. An added benefit that results from using REFPROP is the access to the FORTRAN source code that NIST provides with every REFPROP software license. By directly compiling the REFPROP source code with the PFSAT source code, the computation speed is substantially increased as opposed to simply using the pre-compiled REFPROP DLL. This is primarily because the pre-compiled REFPROP DLL uses an intricate unit converter that is called each time a fluid property is needed. PFSAT has been configured such that this unit converter is no longer needed.

The most significant contribution to the total heat leak in spacecraft propulsion systems arises from radiation heat transfer from the environment. As shown in Fig. 5, PFSAT provides the user with four options to calculate the heat leak through the feed line MLI. The preferred method of calculating heat flux through the MLI is using the modified Lockheed correlation shown in Eq. (5) ${ }^{1}$, which accounts for radiation heat transfer as well as solid conduction between contacting insulation layers and interstitial gas conduction from residual gas between the insulation layers. For the purposes of PFSAT, the Lockheed correlation has been modified in Eq. (5) from its original form by multiplying the resultant heat flux by the degradation factor, $D F$. The degradation factor is used to account for installation inefficiencies. The degradation factor has been determined from the baseline ESTA test bed configuration to be 45 , but it is intended to be updated as more validation data become available in order to provide the best fit of data from various sources.

$$
q_{T}^{\prime \prime}=\left[\frac{C_{S} \cdot \bar{N}^{\text {Exps }}}{N_{s}} \cdot\left(\frac{T_{H}^{2}-T_{C}^{2}}{2}\right)+\frac{C_{R} \cdot \varepsilon_{T R}}{N_{s}} \cdot\left(T_{H}^{4.67}-T_{C}^{4.67}\right)+\frac{C_{G} \cdot P}{N_{s}} \cdot\left(T_{H}^{E x p g}-T_{C}^{E x p g}\right)\right] \cdot D F
$$

Where [define terms]

Depending on the specific MLI configuration being analyzed, the parameters in Eq. (5) take on the values shown in Table 1, which were empirically determined for double-aluminized Mylar shields with water-preconditioned silk net spacers. ${ }^{1}$ Additional shield material selections shown in Table 2 have been made available to the user applying these same parameters with the exception of the shield surface emissivity.

Table 1. Lockheed correlation parameters for double-aluminized Mylar shields.

\begin{tabular}{|c|c|c|c|c|c|c|c|}
\hline Perforation & Atmosphere & Exps & Expg & $\boldsymbol{C}_{\boldsymbol{S}}$ & $\boldsymbol{C}_{\boldsymbol{R}}$ & $\boldsymbol{C}_{\boldsymbol{G}}$ & $\boldsymbol{\varepsilon}_{\boldsymbol{T}}$ \\
\hline Perforated S-604 & Nitrogen & 2.63 & 0.52 & $7.30 \times 10^{-8}$ & $7.07 \times 10^{-10}$ & $1.46 \times 10^{4}$ & 0.043 \\
\hline Perforated S-604 & Helium & 2.63 & 0.26 & $7.30 \times 10^{-8}$ & $7.07 \times 10^{-10}$ & $4.89 \times 10^{4}$ & 0.043 \\
\hline Unperforated & Nitrogen & 2.56 & 0.52 & $8.95 \times 10^{-8}$ & $5.39 \times 10^{-10}$ & $1.46 \times 10^{4}$ & 0.031 \\
\hline Unperforated & Helium & 2.56 & 0.26 & $8.95 \times 10^{-8}$ & $5.39 \times 10^{-10}$ & $4.89 \times 10^{4}$ & 0.031 \\
\hline
\end{tabular}

[Insert new table - Table 2 - Double Goldized Polyimide with $\varepsilon_{T R}=0.03$, Silver with $\varepsilon_{T R}=0.02$ (need to verify values with reference) ]. 
Heat leak from line supports and penetrations can also be a significant contribution to the total heat leak in spacecraft propellant feed systems. The heat leak from both of these contributors is modeled using the Fourier rate equation. The geometry for line supports used in the rate equation is based on the geometry of those installed in the baseline ESTA test bed configuration. A solid model of that line support configuration is shown in Fig. 6. For generic feed systems that implement line sizes other than that used in the baseline test bed, the geometry of the line support is scaled based on the tubing outer diameter. Penetrations are modeled as hollow tubes, as in fill and drain lines or branches in a feed line, for example, or they are modeled as solid rods in the case of instrumentation ports.

The thermodynamic vent system is used to reject heat absorbed by the propellant feed line in order to ensure that subcooled liquid is delivered to the engine valve inlet. This is accomplished through a two step process. First, a very small quantity of propellant at the termination of the feed line is throttled through a Joule-Thomson TVS valve with an extremely small flow area, decreasing its temperature to the saturation point based on the pressure downstream of the TVS valve. It has been determined that the pressure drop in the feed line is very low due to the low TVS flow rates required, so it can be assumed that the user input tank pressure is also the pressure at the TVS valve inlet. Because the user also specifies the tank temperature and the target temperature at the end of the feed line and since throttling across the TVS valve is an isenthalpic process, the enthalpy of the saturated fluid downstream of the valve can be easily determined. The vapor quality of the mixture is determined by the pressure drop across the valve.

The second step of the process to reject heat absorbed by the propellant feed line through the TVS is routing the TVS line back along the feed line to create a counterflow heat exchanger. This removes sensible heat from the feed line propellant via latent heat absorbed and rejected by the two-phase fluid in the TVS line. The heat transfer coefficient for the subcooled fluid flowing in the feed line is determined by the Dittus-Boelter equation. The heat transfer coefficient for the boiling fluid flowing in the TVS line has two components: convection heat transfer and nucleate boiling heat transfer. Of the many boiling heat transfer correlations available, Kandilkar's correlation has been implemented in PFSAT [insert references]. This correlation is dependent on the pressure of the fluid, so the two-phase pressure drop must also be calculated. The separated flow model of Lockhart and Martinelli has been shown to be the most accurate correlation [insert reference].

\section{Conclusion}

The JSC Propulsion Systems Branch has developed PFSAT to rapidly predict heat leak into propellant distribution lines based on insulation type, installation technique, line supports, penetrations, and instrumentation. Based on the computed heat leak, PFSAT will then determine the optimum orifice diameter for an optional thermodynamic vent system to counteract the heat leak into the feed line to ensure temperature constraints at the end of the feed line are met. While some validation has been done using data obtained from the ESTA Cryogenic Feed System Test Bed, the tool has continued to evolve over the past two years with modeling methodologies and parameters being updated as more test data has become available and additional parametric capabilities being added. The information presented here represents PFSAT in close to its final form with a formal release of the tool expected at the end of FY 2011. Forward work that remains to be completed includes finishing the user's guide [insert reference] with detailed code verification documentation and evaluating the complete set of data currently available to show evidence of validation. If funding continues to be available in the future, the tool can be updated to improve its accuracy as additional sources of validation data become available and new capabilities can be added, including the possible integration with an existing tank thermal analysis tool, which can provide a more complete analytical examination of the propulsion system.

\section{Acknowledgments}

The authors would like to extend gratitude to Sue Motil and Terri Tramel, the CFM Project Manager and Deputy Project Manager at Glenn Research Center and Marshall Spaceflight Center, respectively, for providing the funding to support development of PFSAT. The authors would also like to thank Dave Plachta from Glenn Research Center and his summer interns Adam Harden and Mike Nussbaum for their contributions based on their expertise and experience with thermal modeling of cryogenic tanks. Finally, this analytical tool could not have been developed without the valuable data obtained with the help of all the engineers and technicians who have contributed to the Cryogenic Feed System Test Bed at JSC’s Energy Systems Test Area.

\section{References}

${ }^{1}$ Keller, C. W., Cunnington, G. R., and Glassford, A. P., “Thermal Performance of Multilayer Insulations,” Lockheed Missiles \& Space Company, LMSC-D349866, NASA CR-134477, Sunnyvale, CA, 1974. 
${ }^{2}$ Lemmon, E. W., Huber, M. L., and McLinden, M. O., REFPROP, Reference Fluid Thermodynamic and Transport Properties, Software Package, Ver. 8.0, National Institute of Standards and Technology, Boulder, CO, 2007. 